\title{
Extremal charged Vaidya and its near horizon geometry
}

\author{
S. Sadeghian ${ }^{1}$, Azizollah Shafiekhani $^{1,2}$
}

${ }^{1}$ Department of Physics, Faculty of Physics and Chemistry, Alzahra University, Vanak, Tehran, Iran.

${ }^{2}$ School of Physics, Institute for Research in Fundamental Sciences, P.O. Box 19395-5531, Tehran, Iran.

\begin{abstract}
Recently d-dimensional spherically symmetric charged Vaidya black hole solution has been constructed. We observe that this nonstationary solution admits extremal limit and study its near horizon geometry. We show that the symmetry of the near horizon geometry is $\mathrm{SO}(2,1) \times \mathrm{SO}(\mathrm{d}-1)$. Our analysis shows that the theorems for the near horizon geometry of stationary extremal black holes, may be extended to nonstationary cases.
\end{abstract}

Keywords: Nonstationary black hole, Apparent horizon, Near horizon geometry. 